\title{
Pain Medication and Corticosteroid Use in Ankylosing Spondylitis, Psoriatic Arthritis, and Rheumatoid Arthritis in the United States: A Retrospective Observational Study
}

\author{
Theresa Hunter · Chi Nguyen · Julie Birt · Joseph Smith • \\ Mingyang Shan $\cdot$ Hiangkiat Tan $\cdot$ Jeffrey Lisse $\cdot$ Keith Isenberg
}

Received: June 4, 2021 / Accepted: July 1, 2021 / Published online: July 26, 2021

(C) The Author(s) 2021

\section{ABSTRACT}

Objective: We compared pain medication use in patients with ankylosing spondylitis (AS), psoriatic arthritis (PsA), and rheumatoid arthritis (RA) versus matched control over 2 years; a subgroup analysis assessed changes in pain medication use in patients who initiated a biologic during 12 months before and after.

Methods: This was a retrospective observational cohort study using an administrative claims database. Newly diagnosed adult patients with AS, PsA, or RA identified between 1/1/2014 and 7/31/2017 were included. Demographics, baseline characteristics, and pain medication use were described using descriptive statistics. Differences in pain medication use were assessed using McNemar's/Wilcoxon signed-rank test for categorical/continuous variables.

Supplementary Information The online version contains supplementary material available at https:// doi.org/10.1007/s40744-021-00344-6.

T. Hunter $(\varangle) \cdot$ J. Birt · M. Shan · J. Lisse Eli Lilly and Company, 893 S. Delaware Street, Indianapolis, IN 46225, USA

e-mail: hunter_theresa_marie@lilly.com

C. Nguyen · J. Smith $\cdot$ H. Tan

HealthCore Inc., Wilmington, DE, USA

K. Isenberg

Anthem Inc., Indianapolis, IN, USA
Results: The study included 2180 AS, 5681 PsA, and 34,047 RA patients to assess overall pain medication use over 2 years; 188 AS, 921 PsA, and 1599 RA patients were included to assess changes in pain medication use 12 months before and after initiation of biologic. Demographics and baseline characteristics were balanced. In the overall cohort, $74.6 \%$ AS, $75.0 \%$ PsA, and $83.0 \%$ RA patients used any pain medication at baseline versus matched control; pain medications use 2 years after diagnosis date was reported in $73.5 \%$ AS, $74.1 \%$ PsA, and 81.3\% RA patients. Among AS, PsA, and RA patients, use of prescribed NSAIDs (AS: 68.1 vs. 51.1\%; PsA: 51.1 vs. $42.5 \%$; RA: 61.1 vs. $41.5 \%$; $P<0.05$ ), glucocorticoids (AS: 56.4 vs. $41.5 \%$; PsA: 57.4 vs. $46.9 \%$; RA: 88.2 vs. $75.3 \%$; $P<0.05$ ), and opioids (AS: 42.6 vs. $36.2 \%$ [nonsignificant]; PsA: 38.1 vs. $33.8 \%$; RA: 52.0 vs. $40.4 \% ; \quad P<0.05) \quad$ significantly decreased 12 months after biologic initiation versus prior. Conclusions: Use of NSAIDs, glucocorticoids, and opioids are common among patients with AS, PsA, or RA, although the reported use of these co-medications after biologic initiation significantly decreases in the first year of treatment.

Keywords: Pain; Biologics; Corticosteroids; Opioids; Spondyloarthritis; Ankylosing spondylitis; Psoriatic arthritis; Rheumatoid arthritis; United States 


\section{Key Summary Points}

The American College of Rheumatology Pain Management Task Force identified pain as the most important patientreported outcome in rheumatology because of substantial effects on healthrelated quality of life.

Understanding the pain medication usage patterns in patients with ankylosing spondylitis (AS), psoriatic arthritis (PsA), and rheumatoid arthritis (RA) and assessing changes in pain medication use before and after initiation of biologics can help decision-makers in making informed decisions.

Findings from this study suggest that use of NSAIDs, glucocorticoids, and opioids are common among patients with AS, PsA, or RA.

Although pain medication use is common in patients with AS, PsA, and RA, the reported use of these co-medications significantly decreases in the first year of treatment after initiating biologics compared with before.

\section{INTRODUCTION}

Ankylosing spondylitis (AS), psoriatic arthritis (PsA), other forms of spondyloarthritis, and rheumatoid arthritis (RA) fall under chronic inflammatory arthritides that affect large populations worldwide [1]. These conditions are associated with overlapping clinical symptoms, including joint pain (specifically, axial back pain in AS), stiffness, and swelling [2-4]. Disease burdens of AS, PsA, and RA are reported to be similar when assessing patient-reported outcomes (PROs) and disease activity measures [5].

The primary objectives of treatments for AS, PsA, and RA are to maximize long-term quality of life through control of symptoms, mitigation of structural pathological progression, and normalization of function and social participation. The medications for AS, PsA, and RA achieve this by thwarting the overactive immune response and relieving inflammation and pain $[4,6,7]$. The American College of Rheumatology (ACR) Pain Management Task Force (PMTF) identified pain as the most important PRO in rheumatology because of substantial effects on health-related quality of life (HRQoL) $[8,9]$. Treatment planning often focuses on proximal causes, such as tissue injury and inflammation, with the intent of treating local and systemic inflammation [9].

Non-steroidal anti-inflammatory drugs (NSAIDs), glucocorticoids, opioids, and neuromodulators are conventionally used to manage pain and/or inhibit inflammation in AS, PsA, and RA while disease-modifying antirheumatic drugs (DMARDs) slow the progression of the disease by relieving underlying inflammatory responses [4, 6, 8, 10]. Highly specific biologics and targeted synthetic DMARDs are generally prescribed after the failure of conventional synthetic DMARD (csDMARD) therapy in clinical care and have shown to improve HRQoL by reducing inflammatory pain symptoms [11-14]. Biologic DMARDs (bDMARDs) such as adalimumab, etanercept, ixekizumab, secukinumab, golimumab, and the Janus kinase inhibitor tofacitinib are reported to significantly reduce pain in patients with AS, PsA, and/or RA in clinical trials [15-22].

In a prior study, patients with rheumatoid arthritis (RA) who did not receive DMARDs were either receiving treatment with opioids $(56 \%$ overall, 19\% chronic use), pain medications in general (68\%), and glucocorticoid steroids (38\%) [23]. As per the ACR PMTF report, characterization of pain (onset, duration periodicity, and impact on functioning) is vital in creating a comprehensive treatment plan to reduce pain in the management of patients with musculoskeletal disease [9]. However, pain can persist even after achieving remission of inflammatory manifestations in RA [24, 25]. In a Danish cohort study of 107 patients with AS receiving tumor necrosis factor inhibitors, fluctuations of pain and fatigue were noted despite stable disease activity, suggesting that these factors may 
need to be addressed separately in clinical practice [26]. Understanding the pain medication usage patterns and changes in pain medication use before and after initiation of biologics can help inform decision-makers about their impact on pain.

Therefore, we (1) compared overall pain medication use among patients with AS, PsA, and RA over a 2-year period relative to matched controls and (2) evaluated changes in pain medication use in patients with AS, PsA, or RA during the 12-month period before and after initiation of biologics using data from a large administrative claims database.

\section{METHODS}

\section{Study Design and Data Source}

This retrospective analysis was conducted using administrative medical and pharmacy claims from the HealthCore Integrated Research Database (HIRD) ${ }^{\circledR}$ between 1/1/2013 and 7/31/2019. The HIRD ${ }^{\circledR}$ contains clinically and geographically diverse longitudinal claims data from Anthem health insurance plans across the US, representing over 50 million lives. IRB approval was not required as the data was not identifiable.

The study consisted of three cohorts of patients with newly diagnosed AS, PsA, or RA and their matched control of patients without autoimmune disease but with similar demographics and Quan-Charlson Co-morbidity Index (QCI) scores. For assessing the overall pain medication use (NSAIDs, glucocorticoids [oral and intravenous], non-narcotic analgesics, opioids, neuromodulators [antidepressants, anticonvulsants, muscle relaxants], and topical pain medications [diclofenac, capsaicin]), index date was the date of first claim of the disease identified during patient identification period $(1 / 1 / 2014-7 / 31 / 2017)$; patients were followed for 2 years after their index diagnosis for pain medication use and compared to their matched control.

For newly diagnosed cases treated with biologics, the index date was defined as the date of biologic initiation; their pain medication uses during 12 months before and after biologic initiation were examined.

The data in this study were used in full compliance with the relevant provisions of the Health Insurance Portability and Accountability Act. The study was conducted under the research provisions of Privacy Rule (45 CFR Part 160 and Subparts A and E of Part 164) and was exempted from the approval of Institutional Review Board.

\section{Study Population}

The study population included mutually exclusive patient cohorts aged $\geq 18$ years on index date diagnosed with either AS, PsA, or RA. Patients in each of these cohorts were required to have $\geq 2$ claims on different dates within the patient identification period using International Classification of Diseases (ICD; 9th/10th revisions) codes (Supplementary Table S1). Patients with $\geq 1$ year of continuous health plan enrollment before and $\geq 2$ years after the index date (date of first claim of the disease identified during patient identification period) were included in the analysis. Matched control cohorts without any claims for AS, PsA, and RA were identified by matching on age, gender, region of residence, health plan type, and QCI. Control with health plan enrollment around case's index date was identified and the index date for control was set to its matched case. Matched controls were required to have $\geq 1$ year of continuous health plan enrollment before index date; and $\geq 2$ years after index date.

To assess changes in pain medication use during the 12 months before and after initiation of biologics, we included patients with newly diagnosed disease (patients without any index autoimmune disease diagnosis or any DMARD treatment [csDMARD, bDMARD, Janus kinase inhibitors] any time prior to the index date) who met following additional inclusion criteria: (1) $\geq 1$ year of continuous health plan enrollment prior to index date; (2) $\geq 2$ claims for biologics at any time between (and including) index date and end of the study period (not limited to post 12 months period); and (3) $\geq 1$ year of continuous health plan 
enrollment before and after drug initiation date. The biologics and Janus kinase inhibitor considered for this study were as follows:

- AS: adalimumab, certolizumab pegol, etanercept, golimumab, infliximab, secukinumab, and ixekizumab

- PsA: adalimumab, etanercept, infliximab, certolizumab pegol, golimumab, ustekinumab, secukinumab, tofacitinib, ixekizumab, apremilast, and abatacept

- RA: abatacept, adalimumab, anakinra, certolizumab pegol, etanercept, golimumab, infliximab, rituximab, sarilumab, tocilizumab, baricitinib, tofacitinib, and upadacitinib

Patients were excluded from either analysis if they had: (1) $\geq 2$ claims for any non-index autoimmune diseases; (2) multiple autoimmune conditions; (3) $\geq 2$ claims for juvenile idiopathic arthritis; and (4) $\geq 1$ claim for metastatic cancer during the study period.

\section{Study Outcomes}

Baseline patient demographics and clinical characteristics were compared between AS, PsA, and RA patients with their matched control cohorts. The proportion of patients using pain medication at baseline and 2-year follow-up after index diagnosis (dichotomous for each period) were compared. Among patients initiating a biologic on index date, changes in pain medication use 12 months before and after initiating a biologic, and proportion of days covered (PDC [0-1]) for steroids, prescription NSAIDs, and opioids (calculated as number of days covered by a drug days supplied divided by the number of days in the specified time interval, where 1 denotes $100 \%$ adherence) were assessed.

\section{Statistical Analysis}

Demographics, clinical characteristics, and pain medication over a 2-year follow-up after index diagnosis use are described using descriptive statistics. Frequencies and percentages are provided for categorical variables (use of pain medications); means with standard deviations are presented for continuous measures such as PDC. The changes in pain medication use before and after biologic initiation were assessed using McNemar's test; Wilcoxon signed-rank test or paired $t$ test was used for PDC. A conventional alpha of 0.05 and two-tailed level of significance was used in the study. Missing data were reported as missing/unavailable. Statistical analyses were performed in SAS Enterprise Guide 7.15 (SAS Institute Inc, Cary, NC).

\section{RESULTS}

\section{Patient Demographics and Clinical Characteristics on Index Date}

Data from 2180 patients with AS, 5681 patients with PsA, and 34,047 patients with RA were included to describe overall pain medication use over the 2-year period relative to their individually matched cohorts. Baseline demographics and clinical characteristics of these patients are presented in supplementary Table S2. The number of patients and baseline characteristics in the matched controls were generally similar to those in the disease cohorts across all indications (supplementary Table S3).

To assess changes in use of pain medication 12 months before and after initiation of biologics, 188 patients with AS, 921 patients with PsA, and 1599 patients with RA were included in the sub-analysis. Demographics and baseline characteristics of these patients on the index date are shown in Table 1 . In the AS cohort, mean (SD) age was 41.6 (13.2) years, $44.7 \%$ were females, and the mean (SD) time from diagnosis to biologic initiation was 4.0 (7.3) months. In the PsA cohort, mean (SD) age of patients was 48.0 (11.9) years, $50.7 \%$ were females, and mean (SD) time from diagnosis to biologic initiation was 6.6 (8.2) months. The RA cohort was the oldest at 52.3 (12.2) years, had highest proportion of females at $70.5 \%$, and longest mean (SD) time from diagnosis to biologic initiation of 10.2 (9.4) months. The mean (SD) QCI scores in AS, PsA, and RA cohorts were 0.3 (0.7), 0.5 (1.0), and 1.6 (1.1), respectively. A total of $14.9 \%$ patients in AS and $14.4 \%$ in PsA 
Table 1 Demographic and clinical characteristics of patients (who received biologics) with AS, PsA, and RA on index date

\begin{tabular}{|c|c|c|c|}
\hline Variables & AS & PsA & $\mathbf{R A}$ \\
\hline Number of patients, $n$ & $188(100.0)$ & $921(100.0)$ & $1599(100.0)$ \\
\hline \multicolumn{4}{|l|}{ Age on index date (years) } \\
\hline Mean (SD) & $41.6(13.19)$ & $48.0(11.87)$ & $52.3(12.22)$ \\
\hline Female, $n(\%)$ & $84(44.7)$ & $467(50.7)$ & $1127(70.5)$ \\
\hline \multicolumn{4}{|l|}{ Residence region, $n(\%)$} \\
\hline Northeast & $27(14.4)$ & $161(17.5)$ & $215(13.4)$ \\
\hline Midwest & $46(24.5)$ & $220(23.9)$ & $434(27.1)$ \\
\hline South & $73(38.8)$ & $348(37.8)$ & $612(38.3)$ \\
\hline West & $42(22.3)$ & $189(20.5)$ & $330(20.6)$ \\
\hline Other* & $0(0)$ & * & $*$ \\
\hline $\begin{array}{l}\text { Time from index diagnosis to advanced } \\
\text { therapy initiation date (months), mean (SD) }\end{array}$ & $4.0(7.28)$ & $6.6(8.22)$ & $10.2(9.41)$ \\
\hline Quan-Charlson Comorbidity Index, mean (SD) & $0.3(0.68)$ & $0.5(0.96)$ & $1.6(1.07)$ \\
\hline \multicolumn{4}{|l|}{ Quan-Charlson Comorbidity Index categorical, $n$ (\%) } \\
\hline 0 & $148(78.7)$ & $665(72.2)$ & $18(1.1)$ \\
\hline 1 & $28(14.9)$ & $133(14.4)$ & $1,081(67.6)$ \\
\hline 2 & * & $82(8.9)$ & $253(15.8)$ \\
\hline $3+$ & * & $41(4.5)$ & $247(15.4)$ \\
\hline \multicolumn{4}{|l|}{ Baseline conditions of interest, $n(\%)$} \\
\hline Infections & $86(45.7)$ & $470(51.0)$ & $835(52.2)$ \\
\hline Osteoarthritis & $63(33.5)$ & $333(36.2)$ & $817(51.1)$ \\
\hline Hypertension & $47(25.0)$ & $355(38.5)$ & $678(42.4)$ \\
\hline Dyslipidemia & $43(22.9)$ & $297(32.2)$ & $605(37.8)$ \\
\hline
\end{tabular}

$A S$ ankylosing spondylitis, $P_{S A}$ psoriatic arthritis, $R A$ rheumatoid arthritis

${ }^{*}$ Denotes a cell value $\leq 10$ that was blinded for data confidentiality purposes

cohorts had a categorical QCI score of 1. Infections and osteoarthritis were the major baseline comorbidities of interest across all disease cohorts.

\section{Overall Pain Medication Use Over the 2- year Period}

Across all pain medication classes, the use was consistently higher among patients with AS,
PsA, and RA cohorts than the matched controls over the period of 2 years (Fig. $1 \mathrm{~A}-\mathrm{C}$ ). Out of 2180 patients with AS, 5681 patients with PsA, and 34,047 patients with RA, there were 1627 (74.6\%), 4259 (75.0\%), and 28,258 (83.0\%) patients, respectively, who used any pain medication during the baseline period. Pain medications use 2 years after index diagnosis were observed in $73.5,74.1$, and $81.3 \%$ of patients with AS, PsA, and RA, respectively. The most common non-DMARD pain medications used 
A. AS

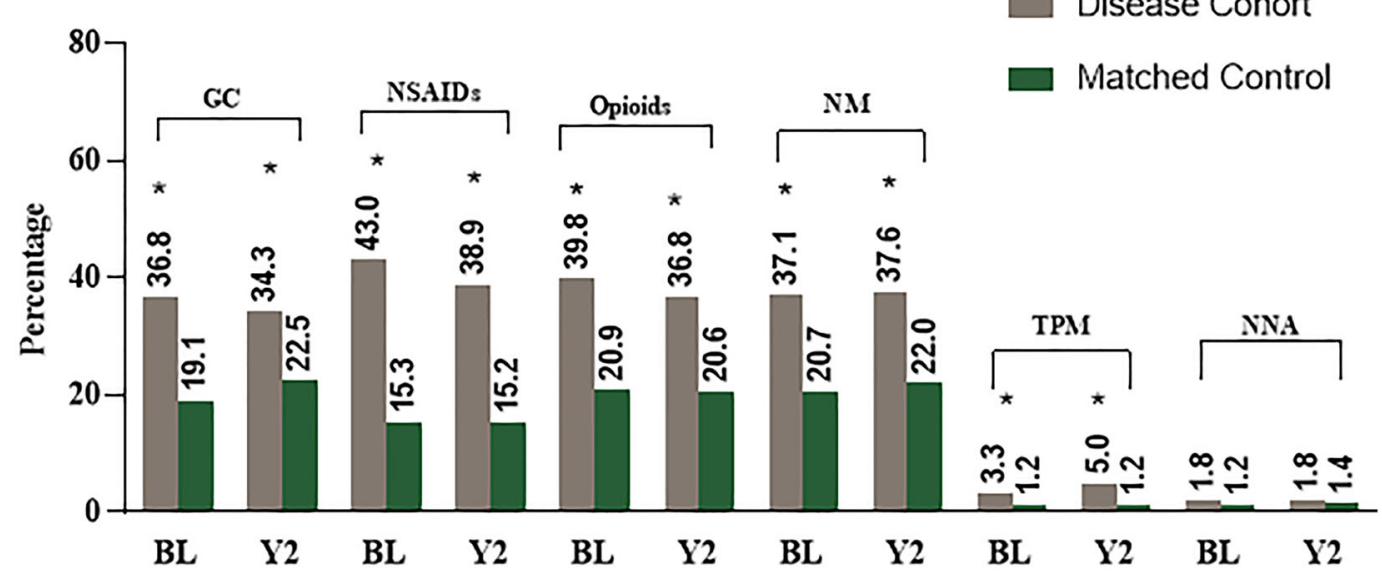

B. PsA

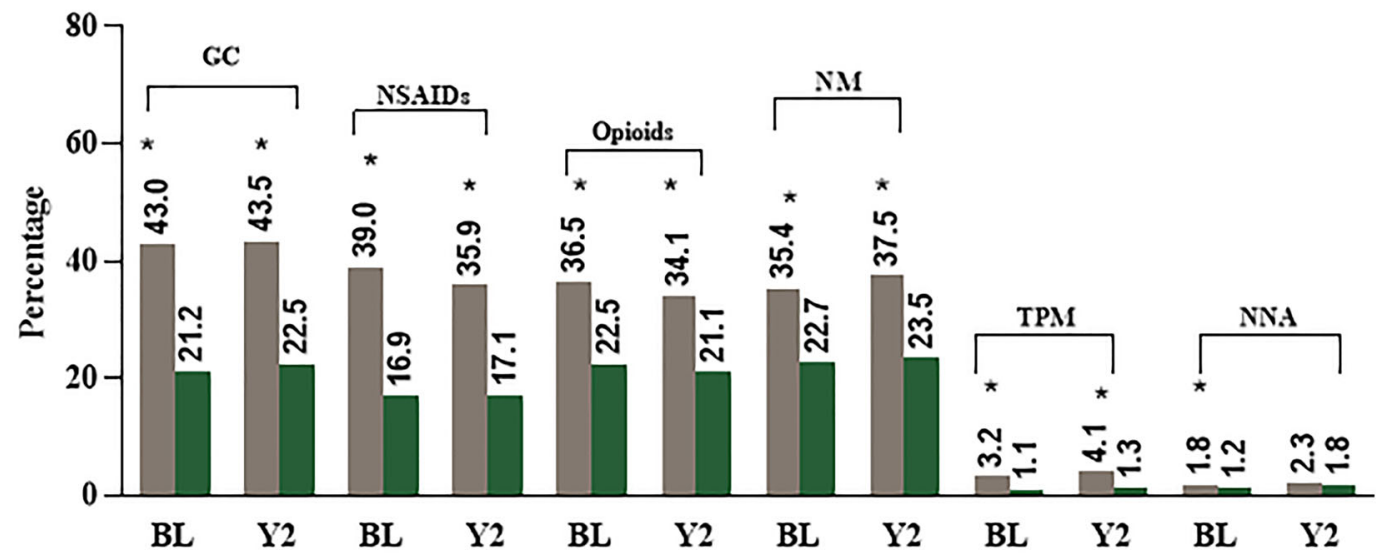

C. RA

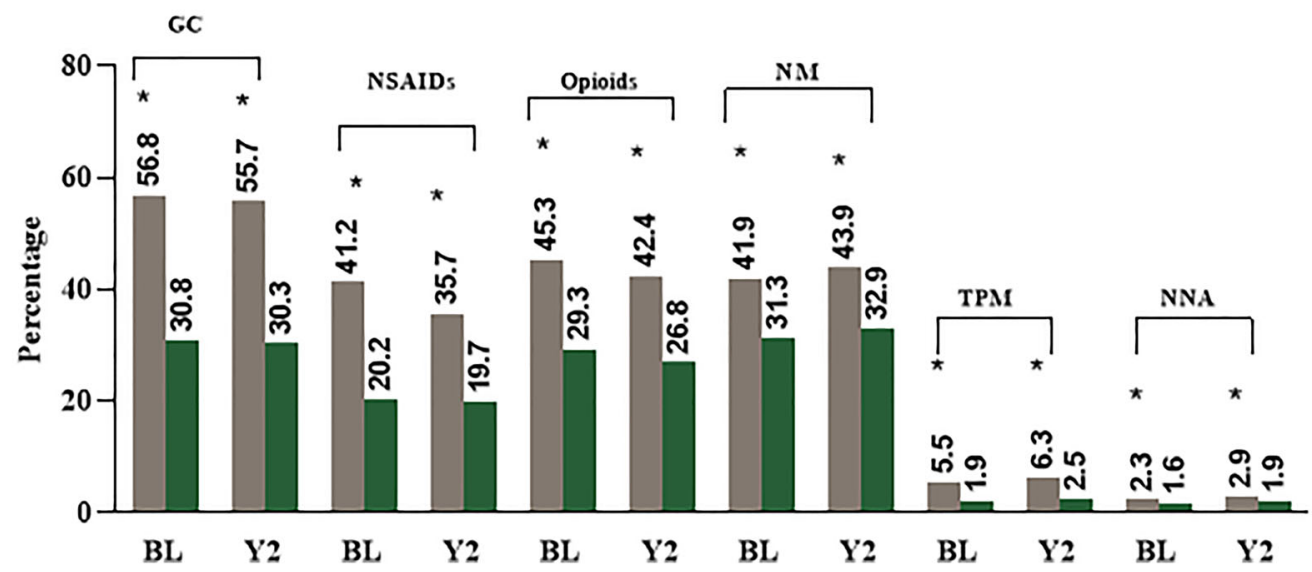

Fig. 1 Overall pain medication use over time among A AS ${ }^{1}, \mathbf{B ~ P s A}{ }^{2}$, and $\mathbf{C ~ R A}{ }^{3}$ patients and matched controls. ${ }^{1}$ For AS cohort/matched control, $N=2180$ each; $P<0.05$ versus matched control. ${ }^{2}$ For PsA cohort/matched control, $N=5681$ each; $P<0.05$ versus matched control. ${ }^{3}$ For RA cohort/matched control, $N=34,047$ each; $P<0.05$ versus matched control. GCs include oral and injectable steroids; NMs include antidepressants, anticonvulsants, and muscle relaxants; TPAs include diclofenac and capsaicin across all cohorts. $A S$ ankylosing spondylitis, $B L$ baseline, $G C$ glucocorticoids, NSAIDs non-steroidal anti-inflammatory drugs, $N N A$ non-narcotic analgesics, $N M$ neuromodulators, $P s A$ psoriatic arthritis, TPM topical pain medication, $R A$ rheumatoid arthritis, $Y 2$ year 2 
for AS, PsA, and RA were either NSAIDs or glucocorticoids (NSAIDs for AS and glucocorticoids for PsA and RA).

\section{Pain Medication Use 12 Months Before and After Initiation of Biologics}

Significantly fewer patients with AS used NSAIDs (68.1 vs. $51.1 \% ; P<0.001)$ and glucocorticoids $\quad(56.4 \quad$ vs. $41.5 \% ; \quad P=0.001)$ 12 months after initiation of biologics compared with before (Fig. 2A). Similarly, in patients with PsA, the use of NSAIDs (51.1 vs. $42.5 \% ; \quad P<0.001)$, glucocorticoids $(57.4$ vs. $46.9 \% ; P<0.001)$, and opioids (38.1 vs. $33.8 \%$; $P=0.013)$ was reduced significantly 12 months after initiation of biologics than before (Fig. 2B). No significant reductions were observed in usage of other pain medication classes in patients with AS and PsA. In patients with RA, the use of NSAIDs (61.1 vs. $41.5 \% ; P<0.001)$, glucocorticoids $(88.2$ vs. $75.3 \% ; P<0.001)$, opioids (52.0 vs. $40.4 \% ; P<0.001$ ), and neuromodulators $(47.4 \quad$ vs. $44.5 \% ; \quad P=0.005)$ significantly decreased 12 months after initiation of biologics (Fig. 2C).

The mean PDC of NSAIDs use 12 months before biologics initiation versus after for AS, PsA, and RA cohorts were 0.39 vs. 0.24; $P<0.001,0.26$ vs. $0.19 ; P<0.001$, and 0.27 vs. $0.19 ; P<0.001$, respectively; similarly, for glucocorticoids use, the values were 0.16 vs. 0.07 ; $P<0.001,0.15$ vs. $0.10 ; P<0.001$, and 0.41 vs. 0.26; $P<0.001$, respectively. The mean PDC of opioids significantly decreased 12 months after initiation of biologics compared to before in the PsA (0.11 vs. $0.09 ; P<0.001)$ and RA (0.15 vs. 0.13; $P<0.001)$ cohorts (Fig. 3).

\section{DISCUSSION}

In this retrospective, observational study representing nationwide data from the US, our findings suggest that the use of pain and antiinflammatory medications (particularly NSAIDs, glucocorticoids, opioids, and neuromodulators) is common in patients with AS, PsA, and RA, even before clinical diagnosis of

\section{A. AS}

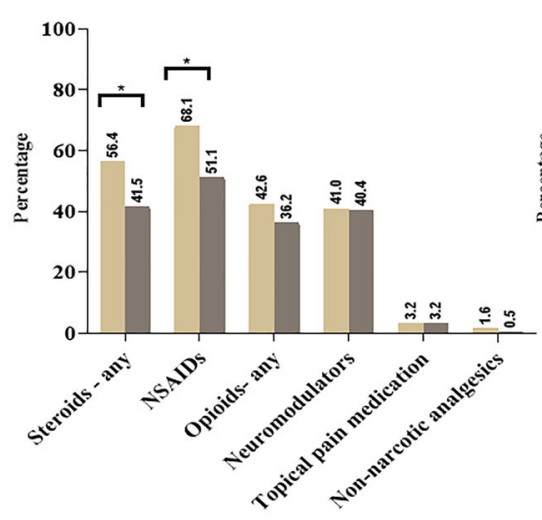

B. PsA

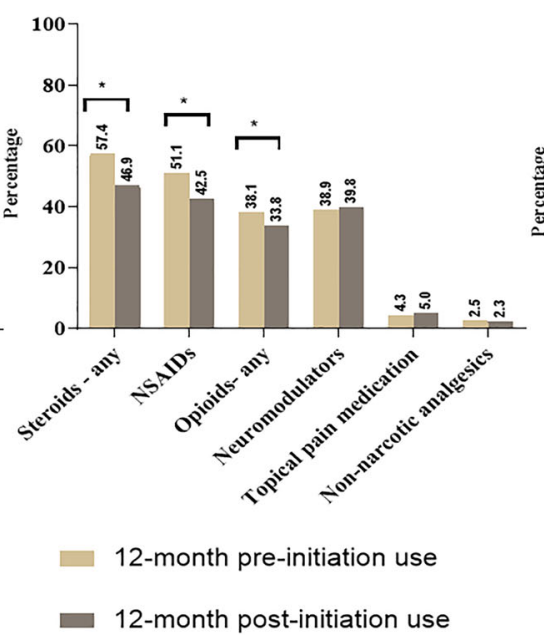

C. RA

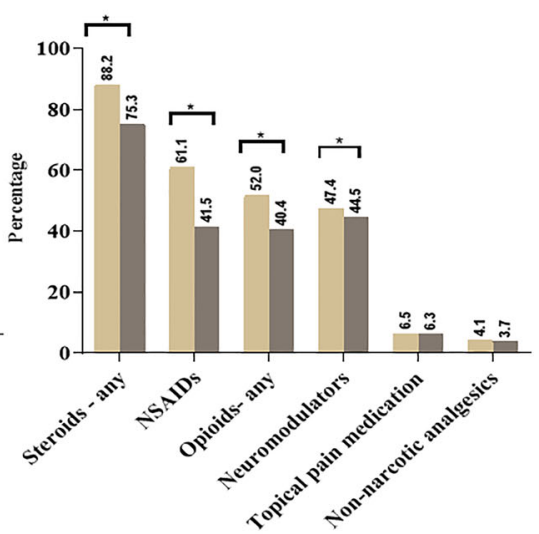

Fig. 2 Changes in pain medication use ${ }^{\#} 12$ months before and after ${ }^{\#}$ biologic initiation in patients with $\mathbf{A} \mathrm{AS}^{1}$, B PsA ${ }^{2}$, and C RA ${ }^{3}$. ${ }^{1}$ For the AS cohort, $N=188$; ${ }^{2}$ For PsA cohort, $N=921$; ${ }^{3}$ For the RA cohort, $N=1599$; ${ }^{*} P<0.05$ was considered statistically significant versus
12 months post-initiation of biologics. "Proportion of patients with $\geq 1$ fills. $A S$ ankylosing spondylitis, NSAIDs non-steroidal anti-inflammatory drugs, $P s A$ psoriatic arthritis, $R A$ rheumatoid arthritis 


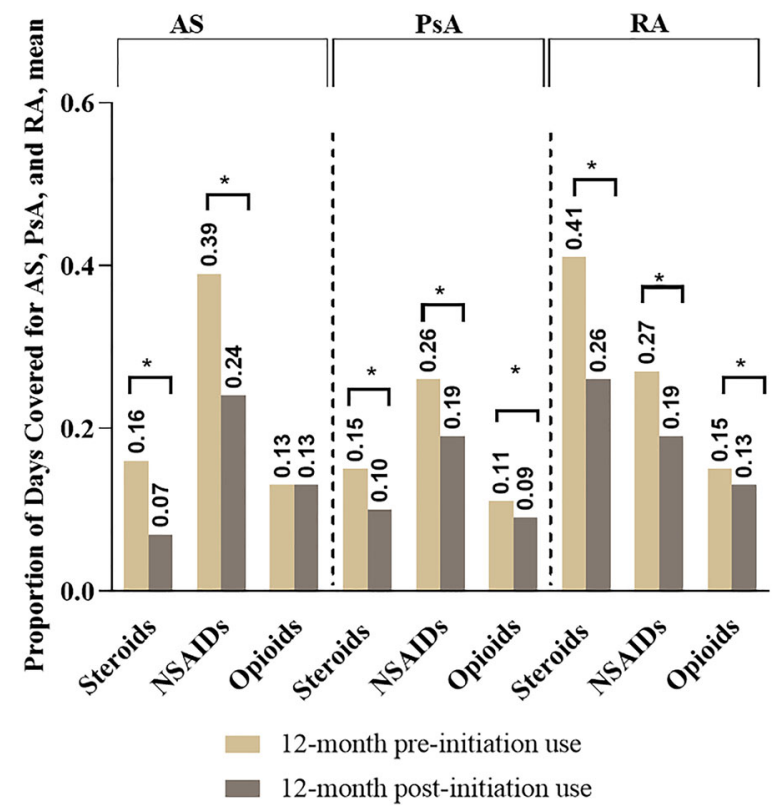

Fig. 3 Proportion of days covered of pain medication use 12 months before and after biologic initiation in patients with A $\mathrm{AS}^{1}, \mathbf{B} \mathrm{PsA}^{2}$, and $\mathbf{C} \mathrm{RA}^{3}$. ${ }^{1}$ For the AS cohort, $N=188 ;{ }^{2}$ For the PsA cohort, $N=921$; ${ }^{3}$ For the RA cohort, $N=1599 ;{ }^{*} P<0.05$ was considered statistically significant versus 12 months post-initiation of biologics. $A S$ ankylosing spondylitis, NSAIDs non-steroidal antiinflammatory drugs, $P s A$ psoriatic arthritis, $R A$ rheumatoid arthritis

these indications. We observed significantly higher pain medication use in patients with AS, PsA, and RA than the matched controls over the period of 2 years. Glucocorticoids, NSAIDs, opioids, and neuromodulators were the most prescribed pain medication classes across all disease cohorts. In patients prescribed a biologic, pain medication use reduced across all disease cohorts 12 months after initiation of biologics compared to before. However, despite reductions, over $30-40 \%$ patients with AS and PsA were still receiving pain medications 12 months after initiation of biologics. Approximately three-fourths of the patients with RA continued using glucocorticoids 12 months after initiation of a biologic. Glucocorticoids, NSAIDs, and opioids were the mostused pain medications across all indications in our study. Our findings are in corroboration with previous reports suggesting the use of NSAIDs and steroids in the management of pain in patients with AS, PsA, and RA [1, 27]. Studies have also shown that long-term prescription of opioids in diseases such as RA and other chronic inflammatory arthritides is common [28, 29].

The use of corticosteroids in pain management in arthritis is common, but often debated. Corticosteroids reduce pain by alleviating inflammation and thus increasing the level of function. However, continuous use of corticosteroids is associated with long-term negative consequences such as immunosuppression, increased risk of infections, ulcers, and osteoporosis to mention a few [30]. Similarly, longterm use of NSAIDs can result in cardiovascular, renal, and gastrointestinal toxicities [31].

Per 'Pain Management Best Practices InterAgency Task Force' convened by the U.S. Department of Health and Human Services together with the U.S. Department of Defense and the U.S. Department of Veteran Affairs with the Office of National Drug Control Policy, the patients with acute and chronic pain in the US face a crisis because of several challenges in getting sufficient care [32]. In 2018, the Centers for Disease Control and Prevention reported that 50 million adults in the US had chronic daily pain [33]. The annual cost of pain to the US was estimated at between $\$ 560$ billion and $\$ 635$ billion [34]. This puts an additional burden on physicians to focus on lowering pain scores while treating patients with acute or chronic pain. Although prescription opioids are used to treat acute and chronic pain in a specific set of patients, the opioid crisis in the US has resulted in overdose deaths associated with prescription opioids [35]. In this context, it is important to understand usage patterns associated with pain medications in real-world situations in patients with inflammatory arthritis.

Other studies have shown that use of pain medication either increases or remains considerably high in patients suffering from musculoskeletal disorders, which often results in acute or chronic pain; a study by Grijalva et al. suggested that despite substantial use of biologics, the use of both NSAIDs and narcotics increased in patients with RA [36]. Another study, using 
the HIRD database, identified a treatment gap in the management of patients with early RA, where a total of 28,306 RA patients did not receive DMARD therapy (though recommended by ACR) during follow-up but received symptomatic treatment such as opioids $(56.5 \%)$ and steroids (37.9\%) [23]. In a trial investigating comparative efficacy of corticosteroid in patients with AS and axial PsA, results suggested that inflammatory back pain in patients with PsA responds significantly better to corticosteroids than in patients with AS [27]. Another study reports that a subset of patients with AS respond well with NSAIDs, however, results from another observational cohort study showed that NSAID intake over time becomes low and treatment adherence decreases despite the persistence of symptoms $[37,38]$. Therefore, it is important to understand usage patterns of pain medications use in the real world to help inform physicians about all the existing options and support them in their decision-making to initiate the most effective treatments.

The usage patterns of pain medications in patients with AS, PsA, and RA suggest that there is need for an effective pain management plan after proper evaluation to establish a diagnosis, with measurable outcomes that focus on improvements, including quality of life, improved functionality, and activities of daily living [32]. A better understanding of other non-pharmacological approaches such as restorative therapy (physiotherapy) and complementary and alternative medicines (acupuncture, massage, yoga, and tai chi, etc.) can also help in possibly reducing heavy pain medication use in AS, PsA, and RA [32].

\section{Strengths and Limitations}

This study used a large and geographically diverse administrative claims database that allowed us to retrospectively analyze the realworld data of disease management. However, the results from this study should be interpreted in the light of a few limitations. Being an observational analysis, information on disease activity, duration of the disease, and clinical rationale could not be obtained to help in further understanding the treatment patterns across disease cohorts. Our study described the burden of pain medications at the aggregate level and does not necessarily reflect individual drug potencies and dosing schedule variations and our study enlists drug categories only and not specific drugs within each category. Also, claims databases do not account for medications obtained from over-the-counter sources. The data in our study are from a patient cohort in the US and derived from an administrative claims database. This may affect its generalizability to populations in other geographies and different health care systems. For assessing pain medication use over the period of 2 years against matched controls, limited medical and disease severity variables were available as covariates to match patients without autoimmune disease with similar medical burden.

\section{CONCLUSIONS}

The use of pain medications such as NSAIDs, glucocorticoids, and opioids are common among patients with AS, PsA, and RA, although the reported use of these co-medications after biologic initiation significantly decreases in the first year of treatment.

\section{ACKNOWLEDGEMENTS}

Funding. The study and the journal's Rapid Service Fee were funded by Eli Lilly and Company, USA.

Medical Writing Assistance. Suchita Dubey, an employee of Eli Lilly Services India Pvt. Ltd. provided writing support.

Authorship. All named authors meet the International Committee of Medical Journal Editors (ICMJE) criteria for authorship for this article, take responsibility for the integrity of the work as a whole, and have given their approval for this version to be published. 
Authors' Contributions. TH, JL, and KI contributed to conception, design, and interpretation of data; $\mathrm{TH}$ also contributed to drafting the manuscript. JB and HT contributed to design and interpretation of the data; HT also contributed to acquisition of the data along with CN. JS and CN were involved in the analysis of the data. All the authors were involved in interpretation of the data and critical revision of the manuscript for intellectual content.

Disclosures. Chi Nguyen, Joseph Smith, and Hiangkiat Tan, are employees of HealthCore, Inc., who received funding from Eli Lilly to conduct this study. Theresa Hunter, Julie Birt, and Jeffrey Lisse are employees and stockholders of Eli Lilly and Company. Mingyang Shan is an employee of Eli Lilly and Company. Keith Isenberg is an employee of Anthem Inc. and owns Anthem stock.

Compliance with Ethics Guidlines. This retrospective analysis was conducted using administrative medical and pharmacy claims from the HealthCore Integrated Research Database. IRB approval was not required as the data was not identifiable.

Data Availability. The datasets analyzed during the current study are not publicly available due to proprietary reasons but are available from HealthCore Inc., USA.

Open Access. This article is licensed under a Creative Commons Attribution-NonCommercial 4.0 International License, which permits any non-commercial use, sharing, adaptation, distribution and reproduction in any medium or format, as long as you give appropriate credit to the original author(s) and the source, provide a link to the Creative Commons licence, and indicate if changes were made. The images or other third party material in this article are included in the article's Creative Commons licence, unless indicated otherwise in a credit line to the material. If material is not included in the article's Creative Commons licence and your intended use is not permitted by statutory regulation or exceeds the permitted use, you will need to obtain permission directly from the copyright holder. To view a copy of this licence, visit http://creativecommons.org/licenses/by$\mathrm{nc} / 4.0 /$.

\section{REFERENCES}

1. Radner H, Ramiro S, Buchbinder R, Landewe RB, van der Heijde D, Aletaha D. Pain management for inflammatory arthritis (rheumatoid arthritis, psoriatic arthritis, ankylosing spondylitis and other spondylarthritis) and gastrointestinal or liver comorbidity. Cochrane Database Syst Rev. 2012;1(1):CD008951.

2. Heiberg T, Kvien TK. Preferences for improved health examined in 1,024 patients with rheumatoid arthritis: pain has highest priority. Arthritis Rheum. 2002;47(4):391-7.

3. Ogdie A, de Wit M, Callis Duffin K, Campbell W, Chau J, Coates LC, et al. Defining outcome measures for psoriatic arthritis: a report from the GRAPPA-OMERACT Working Group. J Rheumatol. 2017;44(5):697-700.

4. van der Heijde D, Ramiro S, Landewe R, Baraliakos X, Van den Bosch F, Sepriano A, et al. 2016 update of the ASAS-EULAR management recommendations for axial spondyloarthritis. Ann Rheum Dis. 2017;76(6):978-91.

5. Michelsen B, Fiane R, Diamantopoulos AP, Soldal DM, Hansen IJ, Sokka T, et al. A comparison of disease burden in rheumatoid arthritis, psoriatic arthritis and axial spondyloarthritis. PLOS ONE. 2015;10(4):e0123582.

6. Singh JA, Saag KG, Bridges SL Jr, Akl EA, Bannuru RR, Sullivan MC, et al. 2015 American College of Rheumatology Guideline for the Treatment of Rheumatoid Arthritis. Arthritis Care Res (Hoboken). 2016;68(1):1-25.

7. Ward MM, Deodhar A, Gensler LS, Dubreuil M, Yu D, Khan MA, et al. 2019 Update of the American College of Rheumatology/Spondylitis Association of America/Spondyloarthritis Research and Treatment Network Recommendations for the Treatment of Ankylosing Spondylitis and Nonradiographic Axial Spondyloarthritis. Arthritis Care Res (Hoboken). 2019;71(10):1285-99.

8. Geenen R, Overman CL, Christensen R, Asenlof P, Capela S, Huisinga KL, et al. EULAR recommendations for the health professional's approach to pain management in inflammatory arthritis and osteoarthritis. Ann Rheum Dis. 2018;77(6): 797-807. 
9. American College of Rheumatology Pain Management Task Force, Report of the American College of Rheumatology Pain Management Task Force. Arthritis Care Res (Hoboken). 2010;62(5):590-9.

10. Zink A, Thiele K, Huscher D, Listing J, Sieper J, Krause A, et al. Healthcare and burden of disease in psoriatic arthritis. A comparison with rheumatoid arthritis and ankylosing spondylitis. J Rheumatol. 2006;33(1):86-90.

11. Zhu W, He X, Cheng K, Zhang L, Chen D, Wang X, et al. Ankylosing spondylitis: etiology, pathogenesis, and treatments. Bone Res. 2019;7:22.

12. Chimenti MS, D'Antonio A, Conigliaro P, Ferrigno S, Vendola A, Ferraioli M, et al. An update for the clinician on biologics for the treatment of psoriatic arthritis. Biologics. 2020;14:53-75.

13. Curtis JR, Singh JA. Use of biologics in rheumatoid arthritis: current and emerging paradigms of care. Clin Ther. 2011;33(6):679-707.

14. Smolen JS, Landewe R, Bijlsma J, Burmester G, Chatzidionysiou $\mathrm{K}$, Dougados $\mathrm{M}$, et al. EULAR recommendations for the management of rheumatoid arthritis with synthetic and biological diseasemodifying antirheumatic drugs: 2016 update. Ann Rheum Dis. 2017;76(6):960-77.

15. Mease PJ, Ory P, Sharp JT, Ritchlin CT, Van den Bosch F, Wellborne F, et al. Adalimumab for longterm treatment of psoriatic arthritis: 2-year data from the Adalimumab Effectiveness in Psoriatic Arthritis Trial (ADEPT). Ann Rheum Dis. 2009;68(5):702-9.

16. Revicki DA, Luo MP, Wordsworth P, Wong RL, Chen N, Davis JC Jr, et al. Adalimumab reduces pain, fatigue, and stiffness in patients with ankylosing spondylitis: results from the adalimumab trial evaluating long-term safety and efficacy for ankylosing spondylitis (ATLAS). J Rheumatol. 2008;35(7):1346-53.

17. Deodhar A, Conaghan PG, Kvien TK, Strand V, Sherif B, Porter B, et al. Secukinumab provides rapid and persistent relief in pain and fatigue symptoms in patients with ankylosing spondylitis irrespective of baseline C-reactive protein levels or prior tumour necrosis factor inhibitor therapy: 2-year data from the MEASURE 2 study. Clin Exp Rheumatol. 2019;37(2):260-9.

18. Orbai AM, De Vlam K, Nash P, Birt J, Gallo G, Stenger K, et al. Fri0357 improved pain and fatigue with ixekizumab treatment in patients with active psoriatic arthritis and previous inadequate response to Tnf inhibitors: three-year follow-up from a phase 3 study (spirit-P2). Ann Rheumatic Dis. 2020;79(Suppl 1):775.1.
19. McInnes IB, Mease PJ, Schett G, Kirkham B, Strand $\mathrm{V}$, Williams $\mathrm{N}$, et al. Secukinumab provides rapid and sustained pain relief in psoriatic arthritis over 2 years: results from the FUTURE 2 study. Arthritis Res Ther. 2018;20(1):113.

20. Hammoudeh M, Zack DJ, Li W, Stewart VM, Koenig AS. Associations between inflammation, nocturnal back pain and fatigue in ankylosing spondylitis and improvements with etanercept therapy. J Int Med Res. 2013;41(4):1150-9.

21. Ogdie A, de Vlam K, McInnes IB, Mease PJ, Baer P, Lukic $\mathrm{T}$, et al. Efficacy of tofacitinib in reducing pain in patients with rheumatoid arthritis, psoriatic arthritis or ankylosing spondylitis. RMD Open. 2020;6(1):e001042.

22. Inman RD, Davis JC Jr, Heijde D, Diekman L, Sieper J, Kim SI, et al. Efficacy and safety of golimumab in patients with ankylosing spondylitis: results of a randomized, double-blind, placebo-controlled, phase III trial. Arthritis Rheum. 2008;58(11): 3402-12.

23. Kern DM, Chang L, Sonawane K, Larmore CJ, Boytsov NN, Quimbo RA, et al. Treatment patterns of newly diagnosed rheumatoid arthritis patients from a commercially insured population. Rheumatol Ther. 2018;5(2):355-69.

24. McWilliams DF, Walsh DA. Factors predicting pain and early discontinuation of tumour necrosis factor-alpha-inhibitors in people with rheumatoid arthritis: results from the British society for rheumatology biologics register. BMC Musculoskelet Disord. 2016;17(1):337.

25. Conaghan PG, Strand V, Alten R, Sullivan E, Blackburn S, Huneault L, et al. OP0107 Pain still remains a high unmet need among psoriatic arthritis patients receiving existing biologic treatment: results from a multi-national real-world survey. Ann Rheum Dis. 2017;76(Suppl 2):96.

26. Madsen OR. Stability of fatigue, pain, patient global assessment and the Bath Ankylosing Spondylitis Functional Index (BASFI) in spondyloarthropathy patients with stable disease according to the Bath Ankylosing Spondylitis Disease Activity Index (BASDAI). Rheumatol Int. 2018;38(3):425-32.

27. Haroon M, Ahmad M, Baig MN, Mason O, Rice J, FitzGerald O. Inflammatory back pain in psoriatic arthritis is significantly more responsive to corticosteroids compared to back pain in ankylosing spondylitis: a prospective, open-labelled, controlled pilot study. Arthritis Res Ther. 2018;20(1):73.

28. Chen SK, Feldman CH, Brill G, Lee YC, Desai RJ, Kim SC. Use of prescription opioids among patients with rheumatic diseases compared to patients with 
hypertension in the USA: a retrospective cohort study. BMJ Open. 2019;9(6):e027495.

29. Whittle SL, Richards BL, van der Heijde DM, Buchbinder R. The efficacy and safety of opioids in inflammatory arthritis: a Cochrane systematic review. J Rheumatol. 2012;90:40.

30. Sparks JA. Rheumatoid Arthritis. Ann Internal Med. 2019;170(1):ITC1-ITC16.

31. Atchison JW, Herndon CM, Rusie E. NSAIDs for musculoskeletal pain management: current perspectives and novel strategies to improve safety. J Manag Care Pharm. 2013;19(9 Suppl A):S3-19.

32. Services USDoHaH. Pain Management Best Practices Inter-Agency Task Force Report: Updates, Gaps, Inconsistencies, and Recommendations 2019. https://www.hhs.gov/sites/default/files/pmtffinal-report-2019-05-23.pdf. Accessed 15 Feb 2021.

33. Dahlhamer J, Lucas J, Zelaya C, Nahin R, Mackey S, DeBar L, et al. Prevalence of chronic pain and highimpact chronic pain among adults-United States, 2016. MMWR Morb Mortal Wkly Rep. 2018;67(36): 1001-6.
34. Smith TJ, Hillner BE. The Cost of Pain. JAMA Netw Open. 2019;2(4):e191532.

35. Ahmad F RL, Spencer M, Warner M, Sutton P. Provisional drug overdose death counts. National Center for Health Statistics 2018. https://www.cdc. gov/nchs/nvss/vsrr/drug-overdose-data.htm. Accessed 15 Feb 2021.

36. Grijalva CG, Chung CP, Stein CM, Mitchel EF Jr, Griffin MR. Changing patterns of medication use in patients with rheumatoid arthritis in a Medicaid population. Rheumatology (Oxford). 2008;47(7): 1061-4.

37. Molto A, Granger B, Wendling D, Dougados $M$, Gossec L. Use of nonsteroidal anti-inflammatory drugs in early axial spondyloarthritis in daily practice: data from the DESIR cohort. Jt Bone Spine. 2017;84(1):79-82.

38. Varkas G, Van den Bosch F. NSAIDs in axial spondyloarthritis: to be continued...? Ann Rheum Dis. 2016;75(8):1423-5. 